\title{
Statin use and mortality in rheumatoid arthritis: an incident user cohort study
}

\author{
Anindit Chhibber, MS; Savannah Hansen, PharmD; and Joseph Biskupiak, PhD, MBA
}

\section{What is already known about this subject}

- Patients with rheumatoid arthritis (RA) often experience complications, such as cardiovascular disease, systemic inflammation, and impaired immune function leading to increased disability, morbidity, and mortality.

- Cardiovascular disease is the leading cause of death in the RA population.

- Guidelines from the American College of Rheumatology are focused on the treatment of RA; however, the risk of all-cause mortality is 1.5 times higher in RA patients than the general population.

\section{ABSTRACT}

BACKGROUND: Patients with rheumatoid arthritis (RA) have higher rates of mortality attributed to the inflammatory nature and the associated burden of cardiovascular complications. Previous research indicates that treatment with statin therapy may play a role in reducing the mortality rate of $\mathrm{RA}$ patients, but similar evidence in U.S. patients is lacking.

OBJECTIVE: To assess the association between statin use and overall mortality among RA patients in the United States.

\section{What this study adds}

- This study demonstrated that RA patients with incident statin use experienced a decreased risk of all-cause mortality when compared with RA patients with no statin use.

- Given the increased mortality risk in RA and contribution of cardiovascular disease to this risk, these findings provide support for raising awareness of the importance of statin therapy among decision makers, health professionals, and people with RA.
METHODS: A population-based study of RA patients with incident statin use was conducted. Patients aged $\geq 18$ years with a diagnosis of RA between January 2007 and December 2015 were included and reviewed for the use of statin medication. Time stratified propensity score matching was used to adequately balance the comparison groups. Logistic regression and Cox proportional hazard models were used to estimate the association.

RESULTS: 19,614 people fulfilled the inclusion criteria for the study out of which 2,089 were statin users. There were 1,883 statin

\section{Author affiliations}

Anindit Chhibber, MS; Savannah Hansen, PharmD; and Joseph Biskupiak, PhD, MBA College of Pharmacy, University of Utah, Salt Lake City.

\section{AUTHOR CORRESPONDENCE:}

Joseph Biskupiak, 801.585.5249;

Joseph.Biskupiak@pharm.utah.edu

J Manag Care Spec Pharm 2021;27(3):296-305

Copyright $\odot 2021$, Academy of Managed Care Pharmacy. All rights reserved.

users that were matched to 1,883 statin nonusers. Baseline characteristics were well balanced across the 2 groups after matching. The hazards ratio for all-cause mortality in patients with RA for statin users was 0.72 (95\% $\mathrm{Cl}=0.56-0.91 ; P=0.008)$ compared with statin nonusers.

CONCLUSIONS: Compared with no use of statins, current statin use is associated with $28 \%$ lower risk of mortality in RA patients. Decision makers and providers should consider and support integration of these results into the current clinical guidelines for delivering quality health care to RA patients.
Rheumatoid arthritis (RA) is a chronic autoimmune disease characterized by an inflammatory polyarthritis that preferentially affects the small joints. ${ }^{1}$ RA is a "multicausal" disease that most likely results from an amalgamation of genetic predisposition and various environmental and lifestyle factors. Patients with this disease often experience complications, such as cardiovascular disease, systemic inflammation, and impaired immune function, which lead to increased disability, morbidity, and mortality. ${ }^{2-4}$ Specifically, patients with RA have been shown to be at a higher risk for comorbidities such as musculoskeletal system diseases, diseases of 
the immune system, endocrine diseases, circulatory system diseases, and diseases of the respiratory system when compared with matched general population. ${ }^{3}$ In addition, when compared with the general population, patients with RA had $54 \%$ higher mortality rates after adjusting for confounders. ${ }^{4}$ The long-term prognosis of patients with RA has improved over the last decades, but survival rates are still lower in RA patients compared with the general population; the gap is thus continuing to widen between these 2 groups. ${ }^{3,4}$

The American College of Rheumatology has established standard guidelines for the treatment of RA, which are targeted at preventing these complications, slowing or preventing disease progression, and managing symptoms through drug therapy. ${ }^{5}$ However, even with current recommendations used in practice, there still remains a risk of all-cause mortality that is 1.5 times higher in the affected population than the general public. ${ }^{4}$ This finding indicates that there is room for improvement in the current practice to incorporate medications that will help reduce this high risk of mortality.

Hydroxymethylglutaryl-coenzyme A reductase inhibitors, more commonly known as statins, have historically been shown to reduce and prevent primary and secondary cardiovascular disease through different mechanisms. ${ }^{6-8}$ In addition to reduction of cardiovascular events, statins have also been observed to decrease risk of mortality by at least $9 \%$ in the general population as evidenced by 3 systematic reviews. ${ }^{9-11}$ This decrease in cardiovascular events and mortality in the general population can be attributed to improved vascular effects, lipid lowering, and anti-inflammatory properties of statins. These 3-pronged benefits of statin therapy lay the groundwork for an expected similar, if not greater, survival benefit endowed on RA patients, as witnessed among the general population.

Previous studies have indicated that RA patients who initiated statin treatment were at a lower risk of all-cause mortality when compared with propensity score-matched controls. Specifically, a population-based cohort study done using a record-linkage database in Scotland reported $57 \%$ lower risk of all-cause mortality $(95 \% \mathrm{CI}=0.20-0.92)$ in statin-exposed RA patients when compared with patients not exposed to statins..$^{12}$ Another population-based longitudinal study of RA patients conducted in Canada assessed the association between discontinuation of statins in RA patients and all-cause mortality. Statin discontinuation, defined as persistent nonuse for $>3$ months during the therapy course, was associated with a higher risk of mortality among RA patients (hazards ratio $[\mathrm{HR}]=1.79$; $95 \%$ $\mathrm{CI}=1.46-2.20$ ) when compared with the counterparts. ${ }^{13}$ In addition, a recent population-based cohort study using data from the United Kingdom indicated that RA patients who initiated statin treatment were at a 21\% lower risk of all-cause mortality when compared with propensity score-matched controls..$^{14}$ Although examining the benefit of statin therapy in patients with RA may not be novel, no studies have tried to elucidate the similar association in the United States, even though 54.4 million U.S. adults suffer from arthritis-about $25 \%$ of the population. Thus, the objective of this study was to further our understanding of the association between statin initiation and all-cause mortality, with a focus on RA patients in the United States.

\section{Methods}

\section{STUDY TYPE AND DATA SOURCE}

This study was a hypothesis-evaluating treatment effectiveness study with a postulation that statins will decrease all-cause mortality in RA patients. This study conformed to the STROBE (STrengthening the Reporting of OBservational studies in Epidemiology) guidelines regarding conduct and reporting of the observational studies.

We carried out a retrospective cohort study using data from the University of Utah Enterprise Data Warehouse (EDW). The EDW, managed and maintained by the University of Utah Health Science Data Resource Center, is the long-term data mart for patient medical, financial, and administrative data. The EDW integrates historical and comprehensive clinical patient records across the University of Utah Healthcare Systems for more than 1.4 million patients. The information recorded includes demographic information; clinical diagnoses, outcomes, and events; laboratory tests; and prescribed drugs. A standardized system of codes involving all possible identifiers assured confidentiality of the study participants. The study was reviewed and approved by the institutional review board at the University of Utah.

\section{STUDY POPULATION}

The study population included patients who had a diagnosis of RA between January 2007 and December 2015 in the EDW. Patients were included if they were aged 18 years or older and had a diagnosis of RA according to International Classification of Diseases, Ninth/Tenth Revision, Clinical Modification (ICD-9/10-CM) codes. Patients were required to have at least 2 outpatient visits or 1 inpatient visit for RA to be eligible. Patients were excluded if they started statin therapy before their RA diagnosis or if their records were missing age, gender, body mass index (BMI), comorbidities, or medication use. 


\section{STUDY DESIGN}

A population-based study of RA patients with incident statin use was conducted. Incident user design was used over prevalent user design to minimize confounding and resulting bias. The prevalent user design includes patients who are already on the treatment before the beginning of the study, whereas incident user design includes new users of the treatment at the start of the study. Incident user design helps alleviate 2 types of bias: (1) prevalent users who have survived the earlier pharmacotherapy, which can cause selection bias, and (2) different covariates for treatment users might be possibly affected by treatment at study initiation. Incident user design ensures that all patient characteristics are measured before treatment initiation and thus dramatically reduces the opportunity for investigators to adjust for variables that are in the causal pathway.

Patients who initiated a statin during an annual block were defined as statin users, while patients who did not initiate a statin were defined as statin nonusers. The initiation date was used as the index date for statin users, and a random date within the 1-year block was assigned as the index date for nonstatin users. Baseline characteristics such as age, gender, BMI, comorbidities, and medication use were assessed within a 1-year time window before the index date. Comparison cohorts were propensity score matched within the same calendar year, leading to 9 annual blocks from January 2007 to December 2015 to account for confounding.

Univariate analysis was conducted to identify the variables eligible for propensity score matching. Propensity scores were estimated separately for each annual accrual block using logistic regression. The variables included in the model were gender, age, BMI, comorbidities, and medication use. Comorbidities included AIDS/HIV, nonmetastatic malignancy, cerebrovascular disease, chronic pulmonary disease, congestive heart failure, dementia, diabetes without complications, diabetes with complications, hemiplegia or paraplegia, metastatic solid tumor malignancy, liver disease, myocardial infarction, peptic ulcer disease, peripheral vascular disease, and rheumatic heart disease. Medications included antihypertensive drugs, diuretics, glucocorticoids, anticoagulants, insulin, nitrates, disease-modifying antirheumatic drugs (DMARDs), and nonsteriodal antiinflammatory drugs (NSAIDs).

For each statin user, we identified a propensity scorematched subject who did not initiate a statin during the accrual block within a caliper range that was equal to one fourth of the standard deviation of the propensity scores. Standardized differences before and after adjustment for propensity score were also calculated, and variables with standardized differences $<0.10$ were considered to be well balanced.

\section{OUTCOME ASSESSMENT}

The primary outcome of interest was all-cause mortality as defined by the date of death recorded in the University of Utah EDW. Logistic regression and Cox proportional regression were used to assess the outcome.

Logistic regression was performed in propensitymatched cohorts to assess the association between statin initiation and all-cause mortality after 3 years of follow-up. To prevent known residual confounding, additional regression adjustments after adjustment of propensity score were also performed.

In addition to logistic regression, Cox proportional hazard models were used to estimate the association of statin initiation with the risk of all-cause mortality across time. Proportionality assumption was tested using Schoenfeld and scaled Schoenfeld residuals. Participants were censored at occurrence of event or at the end of the study period. To prevent known residual confounding, additional regression adjustments after adjustment of propensity scores were also performed, similar to logistic regression. Cumulative hazard plots were generated to estimate time trends.

\section{STATISTICAL ANALYSIS}

Categorical variables were presented as percentages, and continuous variables were presented as means or medians, as appropriate. We analyzed the data using a simulated intention to treat scenario, where subsequent changes in treatment of the participants who used or did not use statins did not modify the category of use. Additional analyses with follow-up time curtailed at 1 year, 2 years, and 3 years for all subjects was performed to account for probable potential for discontinuation of treatment. P values less than 0.05 were considered statistically significant. All statistical analyses were performed using Stata-SE 15 (StataCorp, College Station, TX).

\section{Results}

Between January 2007 and December 2015, 1,286,141 patients were available in University of Utah EDW for the potential inclusion in the study. Of these patients, 19,614 people fulfilled the inclusion criteria (Figure 1). The baseline characteristics of the cohort are summarized in Supplementary Table 1 (available in online article). The mean age of the eligible participants was 51 years, and the majority of the cohort consisted of females (75.08\%). Of those participants included, $17.91 \%$ had pulmonary disease; $10.39 \%$ had diabetes; $9.63 \%$ had liver disease; $5.05 \%$ had renal disease; and 


\section{FIGURE 1 STROBE Patient Flow Diagram}

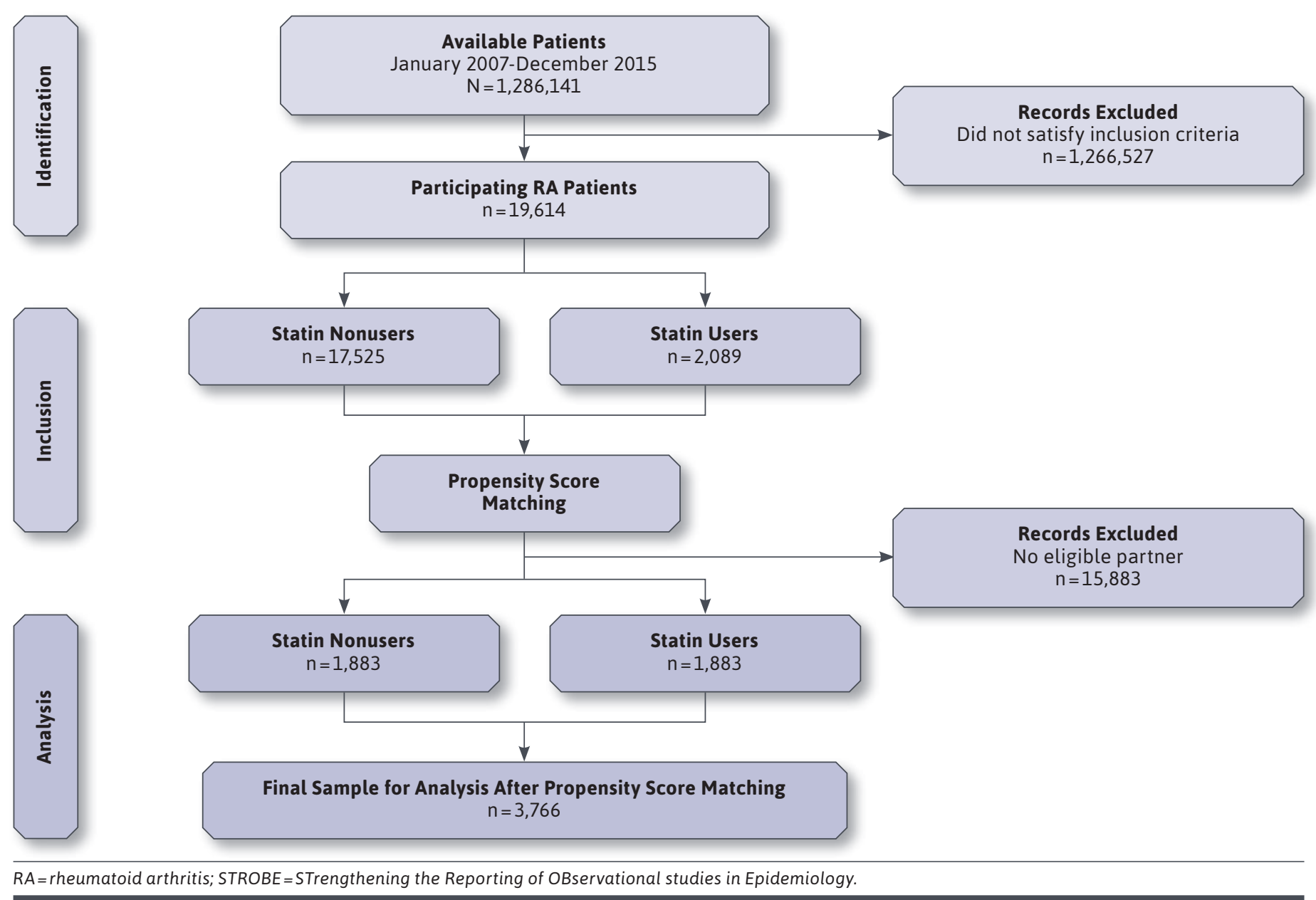

17.64\% had rheumatic heart disease (Supplementary Table 1). In addition, $17.90 \%$ were taking hypertensive drugs; $28.33 \%$ were taking glucocorticoids; $7.34 \%$ were taking insulin; 4.66\% were taking DMARDs; and $28.41 \%$ were taking NSAIDs at baseline (Supplementary Table 1).

Table 1 shows the baseline characteristics for eligible participants stratified by statin initiation before and after 1:1 propensity score matching. Before matching, there were significant differences in baseline characteristics between statin users and nonusers. Compared with nonusers, statin users were older (50.41 years vs. 60.77 years; $P<0.001$ ), had a greater number of males (23.63\% vs. 35.71\%; $\mathrm{P}<0.001)$, and a greater BMI (25.28 vs. 27.88; $\mathrm{P}<0.001)$. In addition, cerebrovascular disease (5.65\% vs. 11.63\%; $\mathrm{P}<0.001)$, congestive heart failure (3.71\% vs. 9.24\%; $\mathrm{P}<0.001)$, dementia (0.55\% vs. $1.20 \%$; $\mathrm{P}<0.001)$, diabetes (8.85\% vs. $23.31 \%$; $\mathrm{P}<0.001)$, paraplegia (1.91\% vs. $2.68 \% ; \mathrm{P}=0.016)$, myocardial infarction $(1.56 \%$ vs. $7.28 ; \mathrm{P}<0.001)$, peripheral vascular disease (4.11\% vs. 10.72\%; $\mathrm{P}<0.001)$, renal disease $(4.48 \%$ vs. 9.86\%; $\mathrm{P}<0.001)$, and rheumatic disease $(17.21 \%$ vs. 21.21\%; $\mathrm{P}<0.001)$ were more prevalent in statin users. Statin nonusers were also significantly different from statin users in the use of antihypertensive drugs (13.86\% vs. $51.80 \%$; $\mathrm{P}<0.001)$, diuretics (9.23\% vs. 32.31\%; $\mathrm{P}<0.001)$, glucocorticoids (26.84\% vs. 40.89\%; $\mathrm{P}<0.001)$, anticoagulants $(4.95 \%$ vs. 14.70\%; $\mathrm{P}<0.001)$, insulin $(5.27 \%$ vs. $24.70 \%$; $P<0.001)$, nitrates (1.52\% vs.11.68\%; P<0.001), DMARDs (4.79\% vs. $3.54 \%$; $\mathrm{P}<0.001)$, and NSAIDs (27.49\% vs. 36.14\%; $\mathrm{P}<0.001)$. 


\section{TABLE 1 Baseline Characteristics of Unmatched and Propensity Score-Matched Cohorts}

\begin{tabular}{|c|c|c|c|c|c|c|}
\hline \multirow[b]{2}{*}{ Baseline Characteristics } & \multicolumn{3}{|c|}{ Unmatched Cohorts $(n=19,614)$} & \multicolumn{3}{|c|}{ Propensity Matched Cohorts $(n=3,766)$} \\
\hline & $\begin{array}{l}\text { Nonusers } \\
\mathbf{n}=\mathbf{1 7 , 5 2 5}\end{array}$ & $\begin{array}{c}\text { Users } \\
n=2,089\end{array}$ & P Value & $\begin{array}{c}\text { Nonusers } \\
\mathrm{n}=\mathbf{1 , 8 8 3}\end{array}$ & $\begin{array}{c}\text { Users } \\
\mathbf{n}=\mathbf{1 , 8 8 3} \\
\end{array}$ & P Value \\
\hline Age in years, mean & 50.41 & 60.77 & $<0.001$ & 60.78 & 60.14 & 0.111 \\
\hline \multicolumn{7}{|l|}{ Gender, \% } \\
\hline Female & 76.37 & 64.29 & \multirow{2}{*}{$<0.001$} & 66.91 & 65.91 & \multirow{2}{*}{0.512} \\
\hline Male & 23.63 & 35.71 & & 33.29 & 34.09 & \\
\hline BMI, mean & 25.28 & 27.88 & $<0.001$ & 27.28 & 27.53 & 0.300 \\
\hline \multicolumn{7}{|l|}{ Comorbidities, \% } \\
\hline AIDS/HIV & 0.34 & 0.29 & 0.710 & 0.32 & 0.27 & 0.763 \\
\hline Nonmetastatic malignancy & 8.48 & 9.29 & 0.213 & 9.77 & 9.24 & 0.578 \\
\hline Cerebrovascular disease & 5.65 & 11.63 & $<0.001$ & 10.04 & 9.88 & 0.870 \\
\hline Chronic pulmonary disease & 17.73 & 19.34 & 0.071 & 20.13 & 18.37 & 0.173 \\
\hline Congestive heart failure & 3.71 & 9.24 & $<0.001$ & 7.97 & 7.33 & 0.462 \\
\hline Dementia & 0.55 & 1.20 & $<0.001$ & 1.22 & 0.90 & 0.340 \\
\hline Diabetes without complications & 5.72 & 15.03 & $<0.001$ & 13.49 & 12.59 & 0.411 \\
\hline Diabetes with complications & 3.13 & 8.28 & $<0.001$ & 7.33 & 7.43 & 0.901 \\
\hline Hemiplegia or paraplegia & 1.91 & 2.68 & 0.016 & 1.86 & 2.39 & 0.258 \\
\hline Metastatic solid tumor malignancy & 3.11 & 2.78 & 0.404 & 2.50 & 2.81 & 0.543 \\
\hline Liver disease & 9.64 & 9.53 & 0.864 & 10.04 & 9.24 & 0.408 \\
\hline Myocardial infarction & 1.56 & 7.28 & $<0.001$ & 5.26 & 4.78 & 0.502 \\
\hline Peptic ulcer disease & 3.51 & 3.83 & 0.463 & 4.20 & 3.72 & 0.452 \\
\hline Peripheral vascular disease & 4.11 & 10.72 & $<0.001$ & 9.51 & 8.87 & 0.498 \\
\hline Renal disease & 4.48 & 9.86 & $<0.001$ & 8.71 & 8.44 & 0.771 \\
\hline Rheumatic disease & 17.21 & 21.21 & $<0.001$ & 19.86 & 20.07 & 0.870 \\
\hline \multicolumn{7}{|l|}{ Medication use, \% } \\
\hline Antihypertensive drugs & 13.86 & 51.80 & $<0.001$ & 46.20 & 47.37 & 0.472 \\
\hline Diuretics & 9.23 & 32.31 & $<0.001$ & 29.21 & 29.05 & 0.914 \\
\hline Glucocorticoids & 26.84 & 40.88 & $<0.001$ & 41.74 & 39.88 & 0.246 \\
\hline Anticoagulants & 4.95 & 14.70 & $<0.001$ & 12.69 & 13.01 & 0.770 \\
\hline Insulin & 5.27 & 24.70 & $<0.001$ & 19.86 & 19.76 & 0.935 \\
\hline Nitrates & 1.52 & 11.68 & $<0.001$ & 7.65 & 7.65 & 1.000 \\
\hline DMARDs & 4.79 & 3.54 & 0.010 & 3.03 & 3.56 & 0.361 \\
\hline NSAIDs & 27.49 & 36.14 & $<0.001$ & 37.12 & 36.06 & 0.499 \\
\hline
\end{tabular}

After propensity score matching with gender, age, BMI, comorbid conditions, and medication use, no statistically significant differences were observed between the statin nonuser and user cohorts (Table 1). Standardized differences before and after adjustment for propensity score were also calculated, and variables with standardized differences
$<0.10$ were considered to be well balanced (Supplementary Table 2, available in online article).

Logistic regression and Cox proportional hazards regression were used to determine the association of statin initiation and all-cause mortality. Table 2 shows the unadjusted and adjusted odds ratios (ORs) for the 


\section{TABLE 2 Unadjusted and Adjusted Odds Ratios for Mortality Associated with Statin Use}

\begin{tabular}{|c|c|c|c|c|c|c|}
\hline \multirow[b]{2}{*}{ Variables } & \multicolumn{3}{|c|}{ Unadjusted Regression } & \multicolumn{3}{|c|}{ Adjusted Regression } \\
\hline & Odds Ratio & $95 \% \mathrm{Cl}$ & P Value & Odds Ratio & $95 \% \mathrm{Cl}$ & P Value \\
\hline Age & 1.03 & $(1.02-1.05)$ & $<0.001$ & 1.03 & $(1.01-1.05)$ & $<0.001$ \\
\hline Gender & 1.37 & $(0.96-1.95)$ & 0.074 & 1.13 & $(0.78-1.65)$ & 0.502 \\
\hline BMI & 0.97 & $(0.95-1.00)$ & 0.066 & 0.97 & $(0.95-1.00)$ & 0.120 \\
\hline AIDS/HIV & 2.70 & $(0.34-21.26)$ & 0.345 & 1.44 & $(0.15-13.48)$ & 0.744 \\
\hline Nonmetastatic malignancy & 1.48 & $(0.89-2.47)$ & 0.125 & 1.11 & $(0.64-1.92)$ & 0.702 \\
\hline Cerebrovascular disease & 0.96 & $(0.53-1.72)$ & 0.897 & 0.49 & $(0.25-0.96)$ & 0.037 \\
\hline Chronic pulmonary disease & 2.16 & $(1.50-3.13)$ & $<0.001$ & 1.53 & $(0.99-2.36)$ & 0.051 \\
\hline Congestive heart failure & 2.32 & $(1.43-3.75)$ & 0.001 & 1.23 & $(0.68-2.21)$ & 0.478 \\
\hline Dementia & 3.95 & $(1.52-10.25)$ & 0.005 & 2.26 & $(0.79-6.50)$ & 0.128 \\
\hline Diabetes without complications & 1.09 & $(0.66-1.79)$ & 0.716 & 0.64 & $(0.36-1.12)$ & 0.122 \\
\hline Diabetes with complications & 1.35 & $(0.75-2.43)$ & 0.311 & 0.53 & $(0.26-1.06)$ & 0.075 \\
\hline Hemiplegia or paraplegia & 1.42 & $(0.51-3.96)$ & 0.494 & 1.43 & $(0.47-4.33)$ & 0.517 \\
\hline Metastatic solid tumor malignancy & 6.15 & $(3.54-10.70)$ & $<0.001$ & 3.96 & $(2.12-7.37)$ & $<0.001$ \\
\hline Liver disease & 2.70 & $(1.76-4.13)$ & $<0.001$ & 2.16 & $(1.32-3.55)$ & $<0.001$ \\
\hline Myocardial infarction & 1.54 & $(0.79-2.98)$ & 0.199 & 0.93 & $(0.44-1.95)$ & 0.854 \\
\hline Peptic ulcer disease & 1.78 & $(0.88-3.57)$ & 0.105 & 1.03 & $(0.47-2.24)$ & 0.924 \\
\hline Peripheral vascular disease & 1.87 & $(1.16-3.02)$ & 0.010 & 0.91 & $(0.52-1.61)$ & 0.769 \\
\hline Renal disease & 2.28 & $(1.43-3.62)$ & $<0.001$ & 1.45 & $(0.83-2.52)$ & 0.184 \\
\hline Rheumatic disease & 1.99 & $(1.38-2.88)$ & $<0.001$ & 1.22 & $(0.81-1.84)$ & 0.338 \\
\hline Antihypertensive drugs & 2.04 & $(1.43-2.92)$ & $<0.001$ & 1.25 & $(0.84-1.87)$ & 0.263 \\
\hline Diuretics & 2.48 & $(1.76-3.51)$ & $<0.001$ & 1.59 & $(1.07-2.38)$ & 0.022 \\
\hline Glucocorticoids & 1.63 & $(1.16-2.31)$ & 0.005 & 1.20 & $(0.82-1.75)$ & 0.335 \\
\hline Anticoagulants & 1.33 & $(0.83-2.13)$ & 0.225 & 0.86 & $(0.51-1.43)$ & 0.569 \\
\hline Insulin & 3.32 & $(2.34-4.72)$ & $<0.001$ & 2.80 & $(1.84-4.26)$ & $<0.001$ \\
\hline Nitrates & 2.32 & $(1.43-3.75)$ & 0.001 & 1.60 & $(0.94-2.74)$ & 0.080 \\
\hline DMARDs & 0.43 & $(0.10-1.76)$ & 0.243 & 0.70 & $(0.16-2.93)$ & 0.627 \\
\hline NSAIDs & 0.92 & $(0.64-1.32)$ & 0.663 & 0.88 & $(0.60-1.29)$ & 0.520 \\
\hline Statins & 0.61 & $(0.43-0.87)$ & 0.007 & 0.65 & $(0.45-0.94)$ & 0.023 \\
\hline
\end{tabular}

Note: Adjusted for age, gender, BMI, comorbidities, and medication use.

$B M I=$ body mass index; $D M A R D=$ disease-modifying antirheumatic drug; NSAID=nonsteroidal anti-inflammatory drug.

2 comparative cohorts after propensity score matching (see Supplementary Table 2, available in online article, for comparison before matching). Specifically, statin users had a $35 \%$ lower risk of mortality in comparison with statin nonusers when the groups were adjusted for residual confounding $(\mathrm{OR}=0.65,95 \% \mathrm{CI}=0.45-0.94 ; \mathrm{P}=0.023)$.

The cumulative hazard estimates for mortality associated with statin use are shown in Figure 2. Specifically, statin users had a significantly lower risk of mortality when compared with statin nonusers. In addition, the results of the unadjusted and adjusted Cox proportional hazards models predicting all-cause mortality are shown in Table 3 (see also Supplementary Table 3, available in online article, for comparison before matching). Similar to logistic regression, statin users had a $28 \%$ lower risk of mortality in comparison with statin nonusers when groups were propensity score matched and adjusted for residual confounding $(\mathrm{HR}=0.72,95 \% \mathrm{CI}=0.56-0.91 ; \mathrm{P}=0.008)$. 


\section{FIGURE 2 Cumulative Hazard Estimates for Mortality Associated with Statin Use}

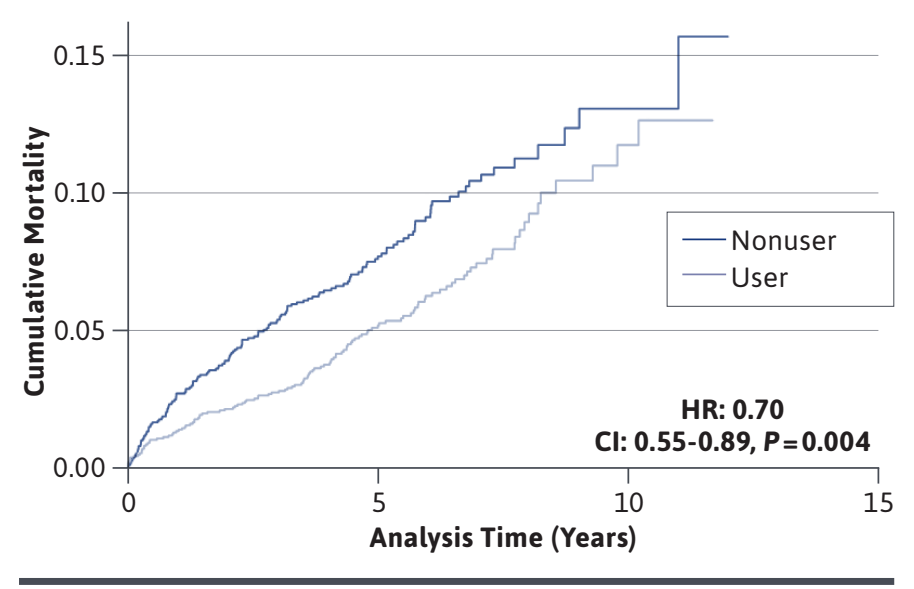

Additional analyses with follow-up time truncated at 1 year, 2 years, and 3 years for all subjects were performed to account for probable potential for discontinuation of treatment. For matched and adjusted cohorts, the HRs in the analyses with follow-up truncated at 1 year, 2 years, and 3 years were $0.66(95 \% \mathrm{CI}=0.39-1.10 ; \mathrm{P}=0.115), 0.71$ (95\% CI $=0.47-1.07 ; P=0.103)$, and $0.63(95 \% \mathrm{CI}=0.44-0.90$; $\mathrm{P}=0.011$ ), respectively (see Supplementary Table 3 , available in online article, for comparison before matching).

\section{Discussion}

In this population-based study of RA patients, we found $28 \%$ lower risk of all-cause mortality among statin users when compared with propensity score-matched statin nonusers (Table 3). The association was evident from the first year of follow-up throughout the succeeding years and was independent of age, gender, BMI, comorbidities, and other medication use (Table 3).

The results of our study align with 3 previous studies that attempted to assess the potential survival effect of statin use in RA patients. Specifically, a population-based cohort study done using a record-linkage database in Scotland reported $57 \%$ lower risk of all-cause mortality (95\% CI $=0.20-0.92)$ in statin-exposed RA patients when compared with statin unexposed patients..$^{12}$ Although these findings were effective, the authors of the study used a prevalent user design as opposed to an incident user design, which might have made the findings vulnerable to selection bias.
Another population-based longitudinal study of RA patient conducted in Canada assessed the association between discontinuation of statins in RA patients and all-cause mortality. Statin discontinuation, defined as persistent nonuse for $>3$ months during the therapy course, was associated with a higher risk of mortality among RA patients $(\mathrm{HR}=1.79 ; 95 \% \mathrm{CI}=1.46-2.20)$ when compared with the counterparts. This association was independent of age, sex, and starting a statin prescription..$^{13}$

In addition, a recent population-based cohort study using data from the United Kingdom found that RA patients who initiated statin treatment were at a $21 \%$ lower risk of all-cause mortality when compared with propensity score-matched controls. ${ }^{14}$ Although examining the benefit of statin therapy in patients with RA may not be novel, our study is the first study to report the beneficial effects of statins in reducing risk of all-cause mortality among RA patients in the United States.

Our findings complement and expand on previous evidence for the beneficial effects of statins to affect multiple steps in the pathogenesis of RA. Thus, a decrease in all-cause mortality in statin users when compared with the counterparts as demonstrated in this study can be attributed to a combined effect of statins in improving vascular function, lowering lipid concentrations and decreasing inflammatory markers in RA patients. More specifically, benefits of statins in RA patients have been thoroughly investigated using vascular function as an endpoint. ${ }^{15-17}$

Administration of simvastatin has been reported to significantly improve "flow mediated vasodilation" in RA patients when compared with placebo. Also, adiponectin along with an improvement in flow mediated vasodilation has been observed when RA patients were given atorvastatin. ${ }^{15}$ The Trial for Atorvastatin in RA (TARA) reported an improvement in vascular risk factors such as ICAM-1 and fibrinogen levels of RA patients after 6 months of treatment with atorvastatin when compared with placebo. ${ }^{17}$

Taking all of this into account, statins seem to have a favorable effect on structural, morphological, and biochemical parameters of vascular function in RA patients. This favorable effect can be a theoretical explanation of the reported benefit of decreased mortality among statin initiating RA patients reported in this study. In addition to the positive effect on the vascular profile in RA patients, statins have also been shown to modulate lipid concentrations. Specifically, nonbiological DMARDs have been reported to decrease the lipid concentrations in RA patients. ${ }^{18}$

Tocilizumab initiation has been associated with a gradual decrease and stabilization of low-density lipoproteins when compared with noninitiators after 2 years of treatment. ${ }^{19}$ In addition, low doses of atorvastatin have also been reported 
TABLE 3 Unadjusted and Adjusted Hazard Ratios for Mortality Associated with Statin Use

\begin{tabular}{l|c|c|c}
\hline Follow-up Time & Hazards Ratio & $95 \%$ CI & P Value \\
\hline Unadjusted \\
\hline 1-year follow-up & 0.64 & $(0.39-1.07)$ & 0.092 \\
\hline 2-year follow-up & 0.68 & $(0.45-1.02)$ & 0.064 \\
\hline 3-year follow-up & 0.60 & $(0.42-0.85)$ & 0.005 \\
\hline Total follow-up & 0.70 & $(0.55-0.89)$ & 0.004 \\
\hline Adjusted & & $(0.39-1.10)$ & 0.115 \\
\hline 1-year follow-up & 0.66 & $(0.47-1.07)$ & 0.103 \\
\hline 2-year follow-up & 0.71 & $(0.44-0.90)$ & 0.011 \\
\hline 3-year follow-up & 0.63 & $(0.56-0.91)$ & 0.008 \\
\hline Total follow-up & 0.72 &
\end{tabular}

Note: Adjusted for age, gender, BMI, comorbidities and medication use. $B M I=$ body mass index .

to decrease lipid levels in RA patients diagnosed with dyslipidemia when therapy is provided with proper control of chronic inflammation and disease activity. ${ }^{20}$ Apolipoprotein $B$ levels have also been reported to decrease when patients were administered 1 year of rosuvastatin treatment in comparison with placebo. ${ }^{21}$ The lipid-lowering properties of statins can be another theoretical explanation of the reported benefit of decreased mortality among statininitiating RA patients reported in this study.

The cardioprotective effects offered by statins in the general population may be accredited to their lipidlowering properties; however, there is evidence that their remedial attributes expand beyond lipid-lowering into anti-inflammatory properties in RA patients. Specifically, atorvastatin has been reported to improve disease activity score and acute phase reactants in RA patients when compared with placebo or when provided in conjunction with DMARDs. ${ }^{17,22}$ Similar to atorvastatin, simvastatin treatment has also been found to reduce serum markers of inflammation in RA patients. ${ }^{23} \mathrm{~A}$ recent meta-analysis highlighted potential inflammation-modulating properties of statins and reported an association of statins with swollen joint count, c-reactive protein, erythrocyte sedimentation rate, and several other clinical parameters of RA. ${ }^{24}$

Although meaningful, most of the studies investigating anti-inflammatory properties of statins had a different primary end point. The patient population involved in most of these studies were on a combination of different immunosuppressant drugs, so any effect on systemic inflammation cannot be attributed solely to statins. Thus, the antiinflammatory properties of statins in RA patients need further investigation. The probable anti-inflammatory effect of statins can be another theoretical explanation of the reported benefit of decreased mortality among statininitiating RA patients in this study.

Thus, we believe that the decrease in all-cause mortality in statin users when compared with their counterparts as demonstrated in this study can be attributable to a combined effect of statins in improving vascular function, lowering lipid concentrations, and decreasing inflammation in RA patients.

\section{STRENGTHS}

A major strength of this study was the inclusion of participants who are often excluded from clinical trials, thus, realizing goals of real-world evidence generation. For instance, the high proportion of women included in our study coincides with the usual higher proportion of women suffering from RA when compared with men. Incident user design was used over prevalent user design to minimize confounding and resulting bias. The prevalent user design includes patients who are already on treatment before the beginning of the study, whereas incident user design includes new users of the treatment at the start of the study. Incident user design helps alleviate 2 types of biases: (1) prevalent users have survived the earlier pharmacotherapy, which can cause selection bias, and (2) different covariates for treatment users might be possibly affected by the treatment at study initiation. Incident user design ensures that all patient characteristics are measured before treatment initiation, thus, dramatically reducing the opportunity for investigators to adjust for variables that are in the causal pathway.

Another strength of this study was large sample size, with diverse clinical and demographic data making results more generalizable across the population. In addition, propensity score matching was used to better compare cohorts and minimize confounding. Comparison cohorts were propensity score matched within the same calendar year to account for confounding by indication and time trends in treatment recommendations. The difference of results in unmatched and unadjusted cohorts when compared with matched and adjusted cohorts revealed that there was significant confounding by indication and that matching and adjusting for confounders was a good approach to handle the said confounding (Figure 2 and Supplementary Figure 2, available in online article).

Logistic regression and Cox proportional hazards regression were employed to determine the association of statin initiation and all-cause mortality in order to corroborate the results from each modelling technique. 


\section{LIMITATIONS}

Our study is subject to limitations inherent in observational studies. There is potential for misclassification of diagnosis, since RA patients were identified using ICD codes, which have high validity for RA patients, but there is still a small chance of misclassification. Previous studies used ICD codes only to identify RA patients and found no difference in outcomes when compared with the other identification methods. ${ }^{14}$

Our results might be susceptible to confounding, since the information from the databases did not allow us to adjust for all known potential confounders. For instance, certain comorbidities of potential interest, such as hyperlipidemia or stable angina, were not included in the analysis. In addition, we cannot exclude the possibility that there was residual or unknown confounding even after matching for known confounders. Also, all individuals with missing information for any variable were excluded from the eligible patient cohort, which could possibly lead to biased results. Further studies can focus on imputation techniques to better control for these missing values. Also, treatment characteristics of the studied cohort, especially low baseline use of DMARDs and NSAIDs, may limit the generalizability of the results to other RA populations.

Several unexpected associations were found between variables, such as insulin use and all-cause mortality, which shows that cause-specific mortality would have been a better endpoint, but we were unable to examine cause-specific mortality, since these data are generally incomplete in the University of Utah EDW. Further studies can focus on causespecific mortality to better assess and evaluate this association.

\section{Conclusions}

Our population-based study of RA patients with incident statin use demonstrated a decreased risk of all-cause mortality associated with statin initiation when compared with the counterparts. Accordingly, we believe that these results provide strong rationale for conducting bigger observational or interventional studies in order to validate the findings. In addition, decision makers and providers should consider these results and support integration of statin use in current clinical guidelines for delivering better quality health care to RA patients.

\section{DISCLOSURES}

No outside funding supported this study. The authors received no financial support for the research, authorship, and/or publication of this article. The authors have no affiliations with or involvement in any organization or entity with any financial interest or nonfinancial interest in the subject matter or materials discussed in this manuscript.

\section{REFERENCES}

1. Centers for Disease Control and Prevention. Rheumatoid arthritis (RA). July 27, 2020. Accessed January 29, 2021. https://www.cdc.gov/arthritis/basics/ rheumatoid-arthritis.html

2. Sokka T, Kautiainen H, Pincus T, et al. Work disability remains a major problem in rheumatoid arthritis in the 2000s: data from 32 countries in the QUEST-RA study. Arthritis Res Ther. 2010;12(2):R42.

3. Løppenthin K, Appel Esbensen B, Østergaard M, Ibsen R, Kjellberg J, Jennum P. Morbidity and mortality in patients with rheumatoid arthritis compared with an age- and sex-matched control population: a nationwide register study. J Comorb. 2019;9:2235042X19853484.
4. van den Hoek J, Boshuizen HC, Roorda LD, et al. Mortality in patients with rheumatoid arthritis: a 15-year prospective cohort study. Rheumatol Int. 2017;37(4):487-93.

5. Singh JA, Saag KG, Bridges SL Jr, Akl EA, et al. 2015 American College of Rheumatology guideline for the treatment of rheumatoid arthritis. Arthritis Care Res (Hoboken). 2016;68(1):1-25.

6. Thavendiranathan P, Bagai A, Brookhart MA, Choudhry NK. Primary prevention of cardiovascular diseases with statin therapy: a meta-analysis of randomized controlled trials. Arch Intern Med. 2006;166(21):2307-13.

7. Liao JK. Rosuvastatin to prevent vascular events in men and women with elevated C-reactive protein. Curr Atheroscler Rep. 2009;11(4):243-44.

8. Chou R, Dana T, Blazina I, Daeges M, Jeanne TL. Statins for prevention of cardiovascular disease in adults: evidence report and systematic review for the U.S. Preventive Services Task Force. JAMA. 2016;316(19):2008-24.

9. Nayak A, Hayen A, Zhu L, et al. Legacy effects of statins on cardiovascular and all-cause mortality: a meta-analysis. BMJ Open. 2018;8(9):e020584.

10. Cholesterol Treatment Trialists Collaborators; Mihaylova B, Emberson J, et al. The effects of lowering LDL cholesterol with statin therapy in people at low risk of vascular disease: meta-analysis of individual data from 27 randomised trials. Lancet. 2012;380(9841):581-90.

11. Taylor F, Huffman MD, Macedo AF, et al. Statins for the primary prevention of cardiovascular disease. Cochrane Database Syst Rev. 2013;(1):CD004816.

12. Sheng X, Murphy MJ, Macdonald TM, Wei L. Effectiveness of statins on total cholesterol and cardiovascular disease and all-cause mortality in osteoarthritis and rheumatoid arthritis. J Rheumatol. 2012;39(1):32-40. 
13. De Vera MA, Choi H, Abrahamowicz M, Kopec J, Lacaille D. Impact of statin discontinuation on mortality in patients with rheumatoid arthritis: a populationbased study. Arthritis Care Res (Hoboken). 2012;64(6):809-16.

14. Schoenfeld SR, Lu L, Rai SK, Seeger JD, Zhang Y, Choi HK. Statin use and mortality in rheumatoid arthritis: a general population-based cohort study. Ann Rheum Dis. 2016;75(7):1315-20.

15. El-Barbary AM, Hussein MS, Rageh EM, Hamouda HE, Wagih AA, Ismail RG. Effect of atorvastatin on inflammation and modification of vascular risk factors in rheumatoid arthritis. J Rheumatol. 2011;38(2):229-35.

16. Hermann F, Forster A, Chenevard R, et al. Simvastatin improves endothelial function in patients with rheumatoid arthritis. J Am Coll Cardiol. 2005;45(3):461-64.

17. McCarey DW, McInnes IB, Madhok R, et al. Trial of Atorvastatin in Rheumatoid Arthritis (TARA): double-blind, randomised placebo-controlled trial. Lancet. 2004;363(9426):2015-21.
18. Georgiadis AN, Papavasiliou EC, Lourida ES, et al. Atherogenic lipid profile is a feature characteristic of patients with early rheumatoid arthritis: effect of early treatment-a prospective, controlled study. Arthritis Res Ther. 2006;8(3):R82.

19. Soubrier M, Pei J, Durand F, Gullestad L, John A. Concomitant use of statins in tocilizumab-treated patients with rheumatoid arthritis: a post hoc analysis. Rheumatol Ther. 2017;4(1):133-49.

20. Akiyama M, Mawatari T, Nakashima Y, et al. Prevalence of dyslipidemia in Japanese patients with rheumatoid arthritis and effects of atorvastatin treatment. Clin Rheumatol. 2015;34(11):1867-75.

21. Tam LS, Li EK, Shang Q, et al. Effects of rosuvastatin on subclinical atherosclerosis and arterial stiffness in rheumatoid arthritis: a randomized controlled pilot trial. Scand J Rheumatol. 2011;40(6):411-21.
22. Mowla K, Rajai E, Ghorbani A, Dargahi-Malamir M, Bahadoram M, Mohammadi S. Effect of atorvastatin on the disease activity and severity of rheumatoid arthritis: double-blind randomized controlled trial. J Clin Diagn Res. 2016;10(5):OC32-6.

23. Tikiz C, Utuk O, Pirildar T, et al. Effects of angiotensin-converting enzyme inhibition and statin treatment on inflammatory markers and endothelial functions in patients with longterm rheumatoid arthritis. J Rheumatol. 2005;32(11):2095-101.

24. Lv S, Liu Y, Zou Z, et al. The impact of statins therapy on disease activity and inflammatory factor in patients with rheumatoid arthritis: a meta-analysis. Clin Exp Rheumatol. 2015;33(1):69-76. 